\section{Revisitando a literatura sobre custo-efetividade e utilidade em saúde}

\author{
Reviewing the literature of cost-effectiveness and \\ cost-utility analysis in health
}

\begin{abstract}
This study aims to contribute to the dissemination of the theoretical foundations for cost-effectiveness and cost-utility analysis. It also provides backing for reflections on the implementation of studies leading to real benefits for both the population and health system management. Taking $a$ historical perspective, and drawing on the work of renowned authors, the study provides an extensive literature review on cost-effectiveness and cost-utility analysis, from the theoretical formulation to the definition of methodological guidelines. The study also highlights the methodological controversies resulting from the diversity of theoretical approaches. As a result, it recommends conducting research on the theoretical foundations, and particularly the position of the extra-welfarists.
\end{abstract}

Cost-Benefit Analysis; Health Management; Review
Leyla Gomes Sancho 1

\section{Introdução}

A riqueza de debates sobre a fundamentação teórica da análise de custo-efetividade e utilidade e, por conseguinte, de suas abordagens, perspectivas e modelos de decisão atrelados às técnicas - mesmo que consagrado e incorporado como um instrumento adicional para a decisão na alocação de recursos em muitos países centrais -, ainda suscita, inclusive no contexto brasileiro, o sentimento de que não é confiável.

Neumann 1, no que tange ao sentimento de incredulidade perante o instrumento, discorre em seu artigo Why Don't Americans Use CostEffectiveness Analysis?, a relutância de médicos e gestores de planos de saúde em utilizá-lo. O argumento, em relação aos médicos, é que estes não foram ensinados a pensar profundamente sobre escassez de recursos (e trade-offs) e pelo fato de que não há protocolos claros para conduzir ou reportar os resultados dos estudos. Já em relação aos gestores, o argumento é que não há recomendação explícita da parte do governo para seu uso e que os planos têm outros processos mais sofisticados para administrar a atenção médica.

No contexto brasileiro, o sentimento fica patente quando Silva 2 afirma que a análise de custo-efetividade ainda é considerada um mero recurso tecnocrático vinculado ao pensamento neoliberal de contenção de gastos no setor, por utilizar critérios de eficiência econômica. Argumento que, ainda segundo a autora, simplifica 
a abordagem sobre a falta de recursos e os problemas da baixa efetividade, eficiência e qualidade dos serviços de saúde no país. Afirmação, entretanto, que não pode ser generalizada, em vista de que, também no nosso contexto, há autores que defendem sua adoção como elemento central de reformas setoriais, como, por exemplo, Costa \& Melo 3 ao fazerem alusão à apropriação do instrumento para a política de contenção de custos.

Diante desse cenário, e coadunando com a idéia de que o instrumental - independente de suas controvérsias metodológicas - é um importante aliado para a avaliação econômica de tecnologias de saúde (e como tal necessita ter uma clara padronização à luz do nosso contexto), se realizou uma revisão de literatura desde sua fundamentação teórica até a formalização de guias metodológicos por distintas organizações internacionais.

Este trabalho tem como objetivo contribuir com a disseminação do conteúdo teórico dessa área do conhecimento e também oferecer subsídios para reflexões no que tange à consecução de estudos que resultem em reais benefícios para a população e, por decorrência, à gestão do sistema de saúde.

\section{Metodologia}

Trata-se de um estudo teórico, resultado de uma ampla pesquisa de revisão da literatura sobre avaliação econômica em saúde - particularmente sobre as técnicas de custo-efetividade e custoutilidade - no tocante à sua fundamentação teórica, às controvérsias metodológicas e aos guias metodológicos. O estudo foi construído sob uma perspectiva histórica e a partir do ponto de vista de renomados autores dessa área do conhecimento.

As bases principais de dados usadas para tanto foram o MEDLINE (PubMed), o SciELO, o LILACS, o ICER (International Center for Economic Research) e o Google. As palavras-chave utilizadas para a busca foram: health economic e/ou health economic assessment e/ou health economic evaluation e/ou cost-effectiveness e/ ou cost-utility e/ou cost-benefit e/ou health economic theory e/ou theoretical foundation e/ou health economic concepts e/ou guidelines e/ou avaliação econômica e/ou custo-efetividade e/ ou custo-utilidade, fundamentação teórica e/ou avaliação econômica em saúde e/ou conceitos e/ou avaliação econômica em saúde e/ou guias metodológicos e/ou diretrizes metodológicas.

Foram selecionados, como critério de inclusão para a revisão, artigos escritos em inglês, es- panhol e português. Não utilizou-se bancos de dissertação de mestrado ou de teses de doutorado sobre o tema, porque o objetivo do estudo foi contemplado com trabalhos realizados por (re)conhecidos autores.

\section{Consolidação da análise de custo-efetividade e custo-utilidade}

A análise de custo-efetividade e custo-utilidade se consolidaram como técnicas predominantes de avaliação econômica em saúde a partir de 1979. Entre os anos de 1966 e 1978, o número de estudos utilizando a técnica de custo-efetividade se equiparou com o de análise realizada por intermédio da técnica de custo-benefício 4,5.

A partir da década de 1990 firmou-se a tendência de padronização de conduta metodológica para os estudos, principalmente os de análise do custo-efetividade. Isso se materializou, em certa medida, com a realização de um painel de especialistas transformado em livro-texto - Gold et al. 6, em 1996, e com a edição, no mesmo ano, do manual teórico-prático de Drummond et al. 7 . É também fruto dessa padronização a formalização de outros guias metodológicos (guidelines) entre os quais se destaca o WHO-CHOICE 8 . Ressaltando-se que, também nessa mesma década, se incorporou a teoria do bem-estar econômico à técnica, originando a análise do custo-utilidade.

Até próximo dos anos 90, no que tange à fundamentação teórica das técnicas, se dizia que, em relação à análise do custo-efetividade, não só era dada pouca atenção à sua fundamentação como também não havia apenas uma fundamentação. O instrumento se consubstancia em uma série de técnicas de otimização (de cunho matemático) que objetivava apenas a alocação de recursos 9 . Mesmo que um dos primeiros estudos sobre custo-efetividade, o de Weinstein \& Stason 10, datado de 1977, apresentasse, se não tão consistente, mas precursora, formulação teórica.

O instrumento, ainda na perspectiva da otimização, seria mais afeito a uma avaliação de eficiência técnica do que à eficiência alocativa. Opinião não compartilhada por Gafni \& Birch 11, que, de forma enfática, refere a razão custo/efetividade sequer responder a uma avaliação de eficiência técnica, em vista desta não avaliar a maximização de benefícios mediante um recurso fixo, nem a minimização de custo para um dado objetivo. Vale destacar que Gafni \& Birch 11, certamente apologistas da análise de custo-benefício, realizaram estudo no qual avaliaram os métodos à luz da teoria do bem-estar econômico.

A análise do custo-utilidade, ao incorporar a função de utilidade - tópico relacionado à teoria 
do bem-estar econômico -, é considerada para alguns autores como mais consistente. Entretanto, para Gafni \& Birch 11 , diante das regras de decisão dispostas na literatura, essa técnica também não contempla a eficiência alocativa. Isso porque a análise do custo-utilidade foca mais os programas individuais, ao invés de uma alocação geral de recursos.

Diante da inexistência de uma publicação formal sobre os princípios que regem as técnicas, Garber \& Phelps 12, em 1997, ao desenharem um modelo, baseado na teoria de utilidade esperada de von Neumann-Morgenstern, e se depararem com algumas questões importantes, tais como: como incluir os custos e benefícios referentes à perda da produtividade; a pertinência de incluir na estimativa os custos médicos futuros durante os anos estendidos pela intervenção médica corrente; o uso de anos de vida incremental como medida de efetividade discriminatória em relação aos idosos e a possibilidade de encontrar o apropriado cut-off da razão de custo-efetividade de outra maneira que não a do resultado da razão da intervenção médica mais comum em uso, aproximam a construção da fundamentação e sistematizam o conhecimento. O modelo (baseado na teoria) veio ao encontro de uma necessidade de resposta no que concerne à questão do ponto de vista/perspectiva a ser adotado nos estudos sobre análise do custo-efetividade - no caso, o da sociedade.

Para os autores a análise não necessariamente leva a uma melhora potencial de Pareto. A questão tem relação direta com a representatividade do Quality Adjusted Life Years (QALY) como preferência/utilidade, ou seja, se o QALY não representa adequadamente a utilidade, não oferece guia efetivo para a melhora do bem-estar. Como também evidenciaram que o critério aplicado no nível individual leva a um consumo ótimo e quando aplicado a uma população estima uma média e não o somatório individual. Isso porque a demanda por QALY varia dentro de uma população. Em outras palavras, ao aplicar uma razão uniforme, alguns indivíduos recebem uma atenção à saúde cujo benefício marginal excede o custo marginal, enquanto ocorre o contrário com os demais indivíduos.

Ainda segundo os autores, o uso da ferramenta em relação à eqüidade e eficiência na provisão de atenção à saúde é mais igualitário do que a da distribuição baseada no mercado (da atenção à saúde). Na perspectiva do bemestar social, a melhora na eqüidade compensa a perda da eficiência de Pareto e, para tanto, talvez seja necessário denotar um alto cut-off para aqueles cuja dotação de utilidade é baixa.
Essa abordagem também foi apropriada por Bleichrodt 13 que, em síntese e sob uma diferente perspectiva, definiu um algoritmo para eqüidade, tanto na perspectiva da utilidade como na condição de saúde (capabilities). O algoritmo tem como base a eqüidade ex-post (distribuição final de QALYs), a eqüidade ex-ante (com justo processo de alocação de QALYs) e a teoria da utilidade multiatributiva, como base para dirimir o trade-off.

Em 1999, alimentando a construção da fundamentação teórica, Garber 14, como um avanço sobre as suas próprias reflexões, pontua a questão sobre as implicações das taxas de preferência no tempo para a variação interpessoal, sobre os ganhos no bem-estar ao se preceder a um cut-off individual e não um uniforme nas populações heterogêneas, e se a análise do custo-efetividade seria um guia eficaz para a maximização do bem-estar.

A partir desse período, a teoria do bem-estar econômico e, particularmente, a teoria da decisão na sua vertente normativa, passam a fundamentar um grande número de estudos. A teoria da decisão apresenta duas vertentes: a descritiva, que tem como objetivo explicar o fenômeno; e a normativa, que formaliza um padrão de correção ou uma norma.

Entretanto, essa fundamentação não responde a algumas questões de cunho ético, dadas as diferentes preferências/utilidades de acordo com o status sócio-econômico dos distintos grupos de uma população e mesmo entre diferentes populações. Essa assertiva é sempre citada (justificativa) por teóricos como uma das debilidades do método - ao não responder às questões relativas às populações heterogêneas.

A despeito do reconhecimento da importância da teoria do bem-estar econômico e dos modelos matemáticos de otimização na fundamentação teórica das análises do custo-efetividade e custo-utilidade, Culyer 15, como um dos percussores, sistematizou uma abordagem teórica para a técnica, decision making approach (DMA).

Entre as diferenças das distintas abordagens, o ponto de maior interesse recai sobre qual perspectiva é valorizado o bem-estar social e, por conseguinte, o ganho em saúde, em vista de um dado orçamento. Mesmo reconhecendo, de antemão, que a teoria neoclássica não encontra no campo da saúde uma condição perfeita de adequação.

Dentre as premissas da teoria não aplicável ao setor, pode-se citar a relativa à barreira de entrada e saída no mercado da atenção à saúde, que não se enquadra no modelo de competição perfeita, aos problemas relativos à assimetria de informação e a certa condição de monopólio, 
em vista do número reduzido de serviços 16 . Outra condição, referida na literatura, é a relativa à questão da representação da preferência (desejo) no que concerne à saúde na modelagem de uma função de bem-estar social que, de acordo com Garber \& Phelps 12, sugere que o QALY não representa adequadamente a utilidade e que a avaliação global do bem-estar social não deve ser baseada apenas na variável saúde.

\section{As abordagens teóricas e seus problemas metodológicos}

Na abordagem welfarista, fundamentada na tradicional e clássica teoria do bem-estar econômico, o ganho em saúde é dado a partir da utilidade, ou melhor, pelo somatório de preferências/utilidades individuais, de acordo com os bens e serviços consumidos. Na abordagem extrawelfarista (DMA), a perspectiva é a maximização da saúde, em que o QALY, como uma medida de resultado, é mais bem representado pelas "capabilities" do que pela utilidade.

As "capabilities", ao introduzir uma ênfase nas oportunidades que os indivíduos têm, são definidas como aquelas segundo as quais os indivíduos "podem fazer" na perspectiva de determinado contexto de escolha social, em detrimento do que "fazem" (functionings). Ou seja, permitem uma autonomia no que se refere ao sistema ético de valores ${ }^{17}$. Sua principal diferença em relação à utilidade (bem-estar econômico) é que as " $\mathrm{Ca}$ pabilities” oferecem uma abordagem multivariada para o bem-estar, possibilitando uma comparação interpessoal mais fácil de acordo com a abordagem ética.

Ainda no que tange às diferenças entre as abordagens, para os welfaristas a atenção à saúde é definida como algo que contribui para o bemestar - a saúde é importante componente para a utilidade individual. Já para os extrawelfaristas, a atenção à saúde é definida como uma contribuição à saúde propriamente dita, enquanto uma política social, que não depende da maximização da função da utilidade individual, ou melhor, para uma melhora no estado de saúde de acordo com os objetivos das pessoas 18 .

Outra diferença entre as abordagens é a que se refere à concepção de necessidade como oposição à demanda, assim como a concepção de saúde ("capabilities") como oposição à utilidade. A necessidade está atrelada ao benefício advindo da atenção à saúde e à demanda, de acordo com a disposição a pagar.

As diferenças entre as abordagens, de cunho metodológico, podem ser observadas na Tabela 1 .

Questões metodológicas em avaliação econômica segundo as abordagens.

\begin{tabular}{|c|c|c|}
\hline Questão & Proposta welfarista & Proposta extrawelfarista (decision-maker) \\
\hline Operacionalização da & Agregação ponderada das preferências & Preferência societal baseada na implícita \\
\hline perspectiva societal & individuais na função do bem-estar & função de bem-estar social \\
\hline Avaliação dos efeitos & Monetarização (willingness to pay & Unidades naturais ou QALY \\
\hline em saúde & ou outro valor da vida) ou QALY como utilidade & (interpretação específica) \\
\hline Avaliação do custo do & Willingness to pay por tempo ganho/ & Método do capital humano \\
\hline tempo/produtividade & método do capital humano & Custos de fricção \\
\hline Avaliação da atenção & Willingness to pay ou salário & Shadow price ou QALY? \\
\hline informal (caregiver) & (shadow price) & \\
\hline Desconto nos custos & Taxa no longo prazo & Taxa no longo prazo \\
\hline \multirow[t]{2}{*}{ Desconto nos efeitos } & Média das preferências de tempo & Preferência de tempo da sociedade/ \\
\hline & individuais para a saúde & governo para a saúde \\
\hline Eqüidade & Trade off entre eqüidade e eficiência é possível & Talvez explicada e ponderada separadamente \\
\hline Ponderação de outras conseqüências & Possível, mas difícil de traduzir em utilidade & Talvez explicada e ponderada separadamente \\
\hline
\end{tabular}

QALY: Quality Adjusted Life Years.

Fonte: Brouwer \& Koopmanschap 47. 


\section{Discutindo as controvérsias metodológicas}

A consecução de um estudo de custo-efetividade/utilidade, que pressupõe um desenho metodológico a partir de sua dimensão, de sua abordagem e sob qual enfoque, apresenta várias possibilidades, as quais, em certa medida, podem ser vinculadas às controvérsias metodológicas.

Em relação à composição da razão custo/efetividade, as principais controvérsias se assentam na parcela dos custos - custo do tempo (produtividade), desconto de custos e os custos futuros. Já na parcela dos efeitos as controvérsias emblemáticas são o desconto nos efeitos e anos de vida incremental. Tendo em comum entre as parcelas a controvérsia relativa à taxa de desconto.

Há também controvérsias sobre o resultado da análise (razão custo/efetividade); considerálo por um cut-off ou por um limiar, ou decorrente da adoção das league table 19. Assim como em relação à variabilidade e a generalisability (possibilidades de generalização). Ressalta-se ainda os potenciais e diferentes tipos e técnicas de escalas para medir o QALY.

\section{No tocante ao efeito}

O QALY, no seu componente qualitativo, se baseia num procedimento no qual é designado um valor de escala compreendido entre 0 (zero) e 1 (um), ou mesmo abaixo de zero (no caso de situações piores que a morte) para distintos estados de saúde. Sendo que esse valor é definido com base em uma técnica econométrica (standart gamble, rating scale, time trade-off etc.) ou em técnica psicométrica (Quality of Well-Being Scale, EuroQol Instrument etc.).

As escalas, independentemente da técnica associada e das dimensões (domínios, função física, emocional, outras) abordadas, e como um nó crítico, apresentam em geral valores diferentes para um mesmo estado de saúde.

Estudo feito por Nord et al. 20 , no qual foi realizada revisão de 15 trabalhos para avaliar a robustez das diversas escalas, usando-se a técnica person trade-off (baseada na preferência revelada), encontrou que 24 dentre 36 valorações foram baseadas no julgamento do autor, ou seja, arbitrária. Esse fato, atrelado a outra inferência do autor, segundo a qual os valores (scores) dependem do tipo de escala, sugeriu os seguintes questionamentos: não há fundamentação teórica consistente no que concerne à formatação dessas escalas e, em decorrência dos diferentes valores (scores), não há como avaliar a robustez tanto das escalas como da técnica a elas atrelada.
O autor acredita que uma das possíveis respostas para essas questões estaria relacionada à maneira como se interpreta o número de QALYs ganhos - se como uma medida de produção (utilidades individuais) ou como uma medida de valor social, fundamentada na lógica da distribuição, ou melhor, na perspectiva da eficiência. Essa última forma de interpretação consubstancia as leagues tables, cuja proposição é a alocação de recursos até a exaustão, de acordo com a ordenação dos resultados, e na qual a eqüidade não é fator relevante.

Publicação de Olsen 21, em 2000, reforça que os estudos de análise do custo-efetividade e custo-utilidade ignoram a diferença em relação à distribuição do total de ganhos em saúde entre os indivíduos - o que se traduz em um tradeoff entre a maximização de QALYs e sua distribuição. Embasado em proposição de Wagstaff (1991), que incorpora na função de bem-estar um parâmetro de "aversão de iniqüidade", o autor questiona como, na prática, se poderia inferir as preferências (distributivas), a partir dos indivíduos, no sentido de ajustar o valor social para os ganhos em saúde 21. Ou seja, se: $v(\mathrm{Pq})=$ $v$ (pQ), quando $\mathrm{PQ}=\mathrm{pQ}$; em que: $v=$ valor social, $\mathrm{P}$ e $\mathrm{p}=$ número de pessoas, $\mathrm{q}$ e $\mathrm{Q}=\mathrm{QALY}$ ganhos por pessoa.

$\mathrm{O}$ autor parte do pressuposto de que intervenções igualmente boas, com idênticos valores sociais, só o são caso tenham a mesma quantidade de QALYs. Caso a questão se relacione ao maior ganho possível, e apenas de ganhos em saúde, os indivíduos são considerados maximizadores de ganhos em saúde. Se a preferência em relação aos ganhos for a da distribuição de pequenos ganhos para muitos indivíduos, estes são considerados dispersores de ganhos. Então $v(\mathrm{Pq})>v(\mathrm{pQ})$, quando $\mathrm{PQ}=\mathrm{pQ}$ e $\mathrm{P}>\mathrm{p}$. E se a preferência for a de grandes ganhos para um pequeno grupo de indivíduos, então estes são concentradores de ganhos. Então $v(\mathrm{Pq})=v(\mathrm{pQ})$, quando $\mathrm{q}<\mathrm{Q}$ e $\mathrm{P}>\mathrm{p}$.

No que tange aos dois últimos atributos, ainda está implícito o que o autor denomina de "efeito limite", no qual um valor insignificante de QALYs ganhos reverte a noção de eqüidade - tendência a concentrar os ganhos em saúde.

Estudo datado de 2003, de Dolan et al. 22, atrelado a uma proposta de protocolo para a avaliação da implementação do National Institute for Clinical Excellence (NICE), faz interessante discussão sobre o valor do QALY para a sociedade. O trabalho é uma revisão sistemática, e objetiva consubstanciar uma proposição de protocolo (guideline) para determinar o valor relativo do QALY de acordo com as várias características da saúde e de seus determinantes. 
O autor, referindo-se a uma preocupação consensualmente disposta na literatura, e em consonância com o pontuado por Nord (1992), explicita ainda o quanto o QALY traz de preocupações quanto à sua eficácia como um direcionador para a decisão sobre a alocação de recursos na área da saúde 22 .

Com base em evidências empíricas, advindas da revisão desses estudos, e tomando como cerne da questão que o valor social de um dado benefício em saúde pode variar de acordo com distintas características, o autor enumera alguns resultados de interesse para a contextualização no que se refere a estudos de análise do custoefetividade e do custo-utilidade. Tendo como a mais importante conclusão dessa revisão o QALY não ser uma medida constante e a simples maximização de QALYs ganhos na população, ou melhor, QALYs de acordo com as preferências não ser a melhor opção como balizador para o caso de recursos limitados.

Os demais resultados de interesse da revisão se referem ao caso de pessoas que desejam dar benefícios aos indivíduos mais saudáveis, caso os em piores condições se enquadrem em estados de saúde cuja medida de resultado esteja acima de um limite (threshold) estabelecido; a discriminação em relação aos idosos; dar baixa prioridade àqueles pacientes que são os responsáveis por sua doença; dar prioridade mais alta aos doentes que tenham dependentes; ser benevolente em relação aos pacientes de classe social mais baixa - com ressalvas para o gênero masculino -; e não ter preferência quanto à distribuição de benefícios, a não ser que estes sejam pequenos, onde a preferência recai na situação em que é preferível dá-los para poucas pessoas.

Partindo dessas evidências, tendo como parâmetro a "aversão da iniqüidade" (como suporte teórico) e ainda tendo como objetivo derivar ponderações (pesos) para a eqüidade de acordo com distintos subgrupos (através das preferências sociais), o autor propõe um modelo que representa as diferentes distribuições de saúde dentro de uma população. O modelo permite calcular o parâmetro, cuja taxa marginal de substituição representa o peso dado para um grupo vis-à-vis a de outro. Ressaltando ainda que, dependendo do número de atributos a serem considerados, os pesos devem ser derivados separadamente. O autor não recomenda o uso de análises com base em cenários conjugados, ou seja, daqueles compostos por diferentes atributos e por distintos níveis dos atributos.

Ainda no que concerne a essa questão, segundo Krauss-Silva 23, hoje os especialistas tendem ao consenso no qual os pesos usados para ajustar anos de vida por qualidade (nos estados de saúde) devem ser baseados em preferências de indivíduos pertencentes a uma amostra aleatória do público em geral e não por subgrupos, ou seja, uma tentativa de dirimir o problema da eqüidade e, em certa medida, o de tratar a heterogeneidade da população.

\section{No tocante ao custo}

A medida da parcela (numerador) de estudos de análise do custo-efetividade e análise do custoutilidade também incorre em controvérsias, principalmente as relacionadas aos custos indiretos e intangíveis. Os custos a serem incorporados em um estudo são todos os relacionados como diretos, indiretos e intangíveis, sejam os advindos da atenção propriamente dita, os do tempo do paciente, os associados ao cuidado e a outros custos associados à doença - transporte etc.

O custo tem como condição fundamental para sua composição a perspectiva/ponto de vista do estudo e a identificação do efeito da intervenção na mudança do estado de saúde. A abrangência dessas condições significa a inclusão e/ou exclusão de recursos a serem identificados, estimados e valorados, assim como se estes (custos) serão contabilizados no numerador ou se representados no denominador. A opção depende do impacto do item no efeito da intervenção 24 .

Num estudo que opte por trabalhar com o QALY como medida de efetividade/utilidade, uma consideração é pertinente: a que se refere ao custo do tempo do paciente que, se trabalhado como custo de oportunidade no tratamento, deve ser incluído no numerador. Se, entretanto, tiver um significativo impacto na qualidade de vida-efetividade (ajustado) vai para o denominador (efetividade).

Os custos de produtividade são associados com a perda ou diminuição da habilidade para o trabalho ou relativo às atividades de lazer (devido à morbidade) e os referentes à perda econômica devido à morte. Em outras palavras, como o valor de produção perdida devido à doença, ou, de forma mais precisa, como valor da perda de riqueza 25 .

Como a perda da produtividade é um custo de difícil mensuração, em vista da complexidade de sua composição - que tem relação direta com a legislação vigente e até mesmo com as normas do seguro social à luz dos contextos nos quais os estudos de análise do custo-efetividade e custoutilidade são realizados -, é usualmente excluída de uma avaliação econômica. Mas, quando incorporada numa análise, pode ser mensurada com base em duas abordagens: os custos de fricção e o relativo ao método do capital humano. 
O custo de produtividade causa impacto no curto prazo (microeconômico e relacionado com a firma) e no médio prazo (macroeconômico e relacionado com a mudança nos custos unitários, com os prêmios de seguro e com a oferta de trabalho). A conseqüência desse impacto é influenciar o rendimento (riqueza) nacional.

O método do custo de fricção surge em meados dos anos 90, em contraposição ao método do capital humano. O método do capital humano estima potencialmente a produção perdida (rendimento bruto) e reflete o investimento na educação, no treinamento e na experiência no trabalho. O custo de fricção é definido como um método no qual o custo incorrido corresponde àquele entre o período de saída do trabalhador doente/incapacitado e o momento em que este trabalhador é substituído - período de fricção. Esse custo tem relação direta com o tempo em que a organização restaura a produção.

O fundamento em que se assenta o método é o da lógica do mercado de trabalho e, como tal, não incorpora na sua valoração, como no caso do método do capital humano, valores que transcendam os objetivos e o modo pelo qual a firma se organiza. O método do capital humano, por exemplo, no caso de morte prematura, considera como custo o incorrido entre o momento do fato (idade do trabalhador) até a idade de aposentadoria. A conseqüência dessa forma de avaliação é a superestimação dos valores.

A controvérsia em relação aos outros tipos de custos indiretos, como os que avaliam sensações subjetivas (dor etc.) e o tempo do cuidado, é que estes não devem ser valorados em termos monetários e incorporados no numerador da razão de custo-efetividade, mas sim como um impacto no estado de saúde e incorporado no denominador da razão. Essa proposição, em grande medida, é a incorporada pela teoria das "capabilities".

Outras questões relativas à avaliação de custo, relevantes e concernentes a um desenho metodológico, são a utilização ou não de custos futuros (os relacionados com a doença, os não relacionados com a doença e as despesas nãomédicas) - quesito de extrema polêmica -, a taxa de desconto e a correção de valores em determinado índice inflacionário.

Os custos futuros são aqueles advindos de doenças ocorridas pelo aumento da expectativa de vida conseqüente à intervenção e não relacionados à doença/intervenção, os que estão relacionados com a doença/intervenção, e os custos não-médicos, relacionados com o prolongamento da vida.

Em geral os estudos de análise do custo-efetividade e do custo-utilidade, quando incluem os custos futuros, só contabilizam os relativos à doença/intervenção sob avaliação, que são de difícil discernimento se balizados à luz dos custos não relacionados à doença 26 . Weinstein \& Fineberg 27 são a favor da contabilização dos custos futuros relacionados e os não-relacionados à doença, mas não consideram as despesas futuras não-médicas. Ou seja, há uma contradição entre o que é ou não é relacionado à doença, assim como qual a melhor forma de estimá-lo.

$\mathrm{O}$ questionamento sobre a pertinência ou não da inclusão dos custos de atenção à saúde efetivados no futuro é embasado por argumentos de acordo com a anuência. Caso a anuência seja positiva, o argumento é que esses são custos que mantêm o indivíduo vivo. Caso seja negativa, o argumento é que outros custos, tais como os relativos à alimentação e lazer, deveriam ser incluídos. E, independente da anuência, deve-se ter claro que os custos futuros refletem o poder real de compra de uma unidade monetária, a qual hoje pode ser maior do que possa vir a ser amanhã 28 .

No que se refere à incorporação ou não desses custos numa análise custo-efetividade, Drummond et al. 29 fazem algumas inferências sobre a questão. Consideram dois fatores determinantes para a decisão sobre a importância de tentar estimá-los: (1) a extensão da necessidade de provisão de atenção nos anos futuros conseqüente ao programa sob avaliação; e (2) disponibilidade de dados.

Há ainda opiniões mais radicais, como a de Mushlin \& Fintor ${ }^{30}$, em relação a intervenções/ programas de screening, quando afirmam que, ao se contabilizar esses custos, se obterá como resultado a alternativa "não fazer nada" como sendo a intervenção mais custo-efetiva.

\section{No tocante à taxa de desconto}

O grande desafio metodológico em relação à medida do denominador da razão custo/efetividade (resultado) de estudos dessa ordem é o relativo ao desconto no efeito. Ainda hoje essa discussão traz aos economistas da saúde um embate sobre o melhor valor para a taxa de desconto e, no caso de benefícios intangíveis como é a saúde, ser ou não pertinente 31,32,33,34,35.

A preferência no tempo é definida como uma taxa marginal de substituição entre a utilidade (presente e futura) e o correspondente fator de correção. Ou seja, a função de desconto dá um valor presente a uma utilidade em algum ponto no tempo.

A teoria econômica não desconhece que os indivíduos não são indiferentes ao tempo, ou seja, adiam custos, mas não benefícios. Assim como 
ela não desconhece que em competição perfeita contabiliza-se o preço como o custo de referência, o qual representa o custo de oportunidade. No setor saúde, em que não existe a perfeição de mercado, os preços nem sempre refletem os custos, os quais devem então ser apropriados pelo seu valor real e não pelos preços pagos pelo serviço.

Existem duas escolas de pensamento e, por conseguinte, dois distintos e opostos pontos de vista. Um, compartilhado por Lipscomb et al. 33, está sedimentado na perspectiva de que a taxa de desconto deve ser a mesma tanto para os custos quanto para os benefícios. Esses autores argumentam que, mesmo reconhecendo que há uma relação crescente dos benefícios ao longo do tempo, não há na literatura uma base sólida de dados empíricos para uma abordagem diferente, e o ajuste na perspectiva da sociedade ainda necessita ser formulado.

A proposição, entretanto, tem como implicações negativas o fato de não responder aos valores da sociedade e não refletir a preferência no tempo dos indivíduos (ou grupos). No caso de programas do tipo promoção à saúde, por exemplo, cujos benefícios ocorrem no longo prazo, pode-se incorrer numa distorção do resultado, levando a uma decisão equivocada.

$\mathrm{O}$ outro ponto de vista sugere que as taxas não devem ser iguais. Sendo que a relativa aos efeitos em saúde deve ser menor que a imputada aos custos. $\mathrm{O}$ argumento dos autores que compartilham dessa opinião, inclusive Gravelle \& Smith ${ }^{34}$, se baseia em fundamentos relativos ao modelo comportamental da escolha individual e no do bem-estar social. Mas que pode, como decorrência do resultado, adiar programas que apresentem benefícios no curto prazo e, concomitante, um alto valor de incremental cost-effectiveness ratio (ICER).

Não faz parte do escopo deste estudo demonstrar esses fundamentos teóricos - inclusive em termos matemáticos -, mas apenas chamar a atenção para o fato de que não só se pode incorporar elementos como o consumo de cuidados médicos, estilo de vida e escolha ocupacional (modelo comportamental), como também incorporar a saúde na modelagem da função de bemestar social. A incorporação da saúde tem como suporte o argumento de que a taxa de crescimento do valor da saúde é uma média ponderada do crescimento direto do valor do efeito em saúde - em termos de utilidade-, da taxa de crescimento do salário e da taxa de crescimento do salário multiplicado pela elasticidade da utilidade marginal do salário. A relação entre saúde e salário está calcada na taxa marginal de substituição, em que há troca de um montante de salário por um montante de saúde (efeitos em saúde), ou ainda, atrelada a um julgamento de valor.

\section{No tocante ao resultado}

Os estudos de análise do custo-efetividade e do custo-utilidade ao serem difundidos e cada vez mais manuseados por uma gama de gestores, principalmente para orientar a alocação de recursos, traz como necessidade a consubstanciação de uma evidência econômica 36 . Ou seja, a possibilidade da transferência dos resultados das avaliações econômicas. Isso porque esses estudos nem sempre podem ser apropriados para uma dada realidade de forma inconteste 37 .

A possibilidade de generalização (generalisability), como uma resposta a esse anseio, é a verificação de até que ponto os resultados de estudos baseados numa população particular e/ou contexto específico podem ser verdades para outras populações e/ou contextos 38 .

Muito se discute sobre possibilidades de generalização dos métodos e de resultados. Mas esse processo tem fracassado, principalmente no sentido de refletir os fundamentos da ciência econômica e da teoria do comportamento do consumidor - fruto da não-validação dos métodos de acordo com distintos cenários 11,39. Segundo Adam et al. 40, há quatro situações que explicitam a variabilidade: não há concordância entre os guias; os guias não especificam como viabilizar as recomendações; as questões particulares quanto à metodologia não são discutidas; e há falta de aquiescência entre os guidelines que, como conseqüência, ainda segundo os autores, torna necessário não só identificar os fatores que podem causar variabilidade, como também avaliar os métodos empregados para tratar esta variabilidade.

No que tange aos estudos de análise do custo-efetividade e do custo-utilidade, a busca pela possibilidade de generalização (generalisability) não está confinada apenas às características dos pacientes ou às relativas à localidade, mas também depende da organização do sistema de atenção à saúde e dos preços dos insumos. E todas essas características são dependentes da sua relação com o tempo (distintos períodos) 41 .

Dentre os fatores mais afeitos à variabilidade, os relativos à questão geográfica são os mais importantes, particularmente os relativos ao paciente, ao clínico, ao sistema de atenção/cuidado à saúde e os sócio-econômicos. São também relevantes os fatores relativos à unidade de custo associado a um recurso particular e à medida de efetividade. E todos sofrem variação ao longo do tempo, principalmente no que concerne a pre- 
ços, recursos usados, práticas clínicas e casesmix.

Em relação ao paciente, a maior fonte de variabilidade é a verificada entre os subgrupos, tanto em termos demográficos quanto em termos clínicos.

No que concerne à questão demográfica/epidemiológica, fundamental para os estudos, a relevância se inscreve na diferença na incidência e prevalência de uma dada doença nos distintos subgrupos, dado que gera diferentes cases-mix entre localidades.

Já em relação ao clínico (médico), a partir de sua experiência, de sua crença e hábitos, essa variabilidade pode influenciar na efetividade e, por conseguinte, no custo-efetividade 42 . Fato que pode decorrer do modus como o sistema do cuidado incentiva o médico, ou melhor, que depende do tipo de relação entre o paciente e o médico. Exemplo concreto é a demanda induzida pela oferta.

O sistema de atenção/cuidado à saúde tem como principais elementos de variabilidade os relativos aos custos do insumo, os quais, segundo a teoria econômica, são influenciados pela tecnologia, pela taxa de substituição entre trabalho e capital, pelos diferentes insumos usados na produção (e o conseqüente custo) e pelo nível de produtividade. Questão crucial dentro de um país extenso como o nosso 43 .

Outra questão importante que concerne ao sistema de atenção à saúde é a relacionada à vontade de prover recursos adicionais para uma dada unidade de ganho em saúde. De outra forma, o custo de oportunidade que pode ser alto ao se investir numa nova tecnologia.

Em relação aos fatores sócio-econômicos, o elemento-chave é a preferência/utilidade que, de acordo com a localidade, pode impactar o cálculo do QALY e o resultado do estudo.

Outras questões têm sido relatadas na literatura como importantes e necessárias no que se refere aos fatores e suas características. Mas chamaria a atenção para a avaliação das possíveis diferentes funções de produção da(s) alternativa(s) sob estudo nas várias localidades.

De acordo com as questões acima apresentadas, diversos métodos (em geral estatísticos) têm sido utilizados no sentido de avaliar a variabilidade e assegurar a possibilidade de generalização (generalisability) dos estudos. Entretanto, um primeiro alerta a ser evidenciado é o relativo às fontes e parâmetros. Quando existem muitas fontes de dados para um parâmetro é dever do analista distinguir o parâmetro incerto da variabilidade ou heterogeneidade.

Dentre os métodos para avaliar a variabilidade, principalmente naqueles baseados em en- saios clínicos, o da regressão 41 é o mais utilizado. Outros também têm sido empregados, como a análise one-way variance, o teste da homogeneidade etc. Segundo Sculpher et al. ${ }^{41}$, para os estudos realizados com base em modelos de decisão, nos quais os dados não estão diretamente disponíveis e requerem opiniões de especialistas, a abordagem bayesiana pode ser adotada. $\mathrm{O}$ modelo bayesiano, em relação aos parâmetros à luz de distintos períodos, é também o método preconizado.

O modelo multinível, adotado nos estudos do tipo ensaio clínico, facilita a correção de estimativas quanto ao resultado incerto dos estudos de análise do custo-efetividade e análise do custoutilidade, decompondo os efeitos tanto no nível do paciente como em relação à localidade 44 .

Outra maneira de verificar é a apropriação de revisões sistemáticas sobre avaliação econômica que, em certa medida, analisa a consistência de resultados entre distintas localidades.

\section{Considerações finais}

Assim como se reforça a necessidade de se implementar pesquisas no sentido de elucidar o significado e a possibilidade de generalização (generalisability) dos resultados (razão custo/ efetividade) - a propósito desta questão destaca-se a proposta do WHO-CHOICE e da revisão bibliográfica Generalisability in Economic Evaluation Studies in Healthcare: A Review and Cases Studies, editada pela Health Technology Assessment, no ano de 200445 -, na década de 90 já se chamava a atenção para os esforços em relação à padronização e conduta metodológica. Vários foram os guias elaborados no período, como o Australian Pharmaeconomics, o European Community Concerted Action on the Harmonisation of the Methodology for Economic Evaluation of Health Technology e o Panel on Cost-Effectiveness in Health and Medicine.

Dentre os inúmeros guias publicados nos últimos anos o de maior visibilidade, até mesmo por sua aplicabilidade, é o NICE, que subsidia a alocação de recursos do National Health System (NHS) no Reino Unido.

Recentemente no Brasil, a partir de louvável iniciativa do Ministério da Saúde por meio do Departamento de Ciência e Tecnologia, atrelado à Secretaria de Ciência e Tecnologia, foi elaborado um manual de diretrizes metodológicas para a execução de estudos sobre avaliação econômica em saúde 46. No entanto, se avaliado de forma minuciosa, esse incorre em ponto citado por Adam et al. 40, o de não especificar detalhadamente como viabilizar as recomendações. 
É provável, como hipótese, que o uso de recomendações nos guias tenha uma estreita relação com o fato da fundamentação teórica da avaliação econômica em saúde ainda ser um objeto em construção. Nos últimos anos, mais do que reflexões sobre a fundamentação teórica, a tônica dos estudos se atém a construções dos modelos de decisão. Não só pela incorporação de modelos complexos matemáticos e/ou epidemiológico (estatístico), mas particularmente pela incorporação do instrumental da informática que não só permite a complexificação da modelagem dos estudos, como também responde à questão relativa à incerteza das estimativas dos parâmetros utilizados nos estudos.

Nessa medida, e como uma recomendação, sugere-se que a fundamentação teórica seja (re)visitada, particularmente a da vertente extrawelfarista (DMA), de modo que esta possa consolidar a base da avaliação econômica em saúde e que, por conseguinte, possibilite a formatação de guia metodológico mais específico quanto à sua viabilização.

\section{Resumo}

Este trabalho tem por finalidade contribuir com a disseminação do conteúdo teórico desta área do conhecimento, assim como oferecer subsídios para reflexões no que tange à consecução de estudos, os quais resultem em reais benefícios para a população e a gestão do sistema de saúde. Para tanto se realizou, sob uma perspectiva histórica e com base no ponto de vista de reconhecidos autores, ampla revisão da literatura que abrangeu desde sua fundamentação teórica até a formalização de guias metodológicos. O estudo ressalta, inclusive, as controvérsias metodológicas conseqüentes da diversidade das abordagens teóricas. E, como decorrência, recomenda a realização de pesquisas sobre a fundamentação teórica, particularmente a abordada pelos extrawelfaristas.

Análise Custo-Benefício; Gestão em Saúde; Revisão 


\section{Referências}

1. Neumann PJ. Why don't Americans use cost-effectiveness analysis? Am J Manag Care 2004; 10: 308-12.

2. Silva LK. Avaliação tecnológica e análise custo-efetividade em saúde: a incorporação de tecnologias e a produção de diretrizes clínicas para o SUS. Ciênc Saúde Coletiva 2003; 8:501-20.

3. Costa NR, Melo MA. Reforma do Estado e as mudanças organizacionais no setor saúde. Ciênc Saúde Coletiva 1998; 3:52-67.

4. Elixhauser A, Luce BR, Taylor WR, Reblando J. Health care CBA/CEA: an update on the growth and composition of the literature. Med Care 1993; 31(7 Suppl):JS1-11, JS18-149.

5. Elixhauser A, Halpern M, Schmier J, Luce BR. Health care CBA and CEA from 1991 to 1996: an updated bibliography. Med Care 1998; 36 (5 Suppl): MS1-9, MS18-147.

6. Gold MR, Siegel JE, Russel LB, Weinstein MC, editors. Cost-effectiveness in health and medicine. New York: Oxford University Press; 1996.

7. Drummond MF, Stoddart G, Torrance G. Methods for economic evaluation of health care programmes. New York: Oxford University Press; 1996.

8. World Health Organization. Development of WHO guidelines on generalized cost-effectiveness analysis. Geneva: World Health Organization; 2000.

9. Garber AM, Weinstein MC, Torrance GW, Kamet MS. Theoretical foundation of cost-effectiveness analysis. In: Gold MR, Siegel JE, Russel LB, Weinstein MC, editors. Cost-effectiveness in health and medicine. New York: Oxford University Press; 1996. p. 25-53.

10. Weinstein MC, Stason WB. Foudation of cost-effectiveness analysis for health and medical practices. N Engl J Med 1977; 296:716-21.

11. Gafni A, Birsch S. Cost-effectiveness/utility analysis. J Health Econ 1992; 11:279-96.

12. Garber AM, Phelps CE. Economics foundations of cost-efffectiveness analysis. J Health Econ 1997; 16:1-31.

13. Bleichrodt H. Health utility indices and equity considerations. J Health Econ 1997; 16:65-91.

14. Garber AM. Advances in cost-effectiveness analysis of health interventions. Cambridge: National Bureau of Economic Research; 1999. (NBER Working Paper, 7198).

15. Culyer AJ. The normative economics of health care finance and provision. In: McGuirre PF, editor. Provinding health care: the economics of alternative system of finance and delivery. Oxford: Oxford University Press; 1991. p. 65-98.

16. Folland S, Goodman AC, Stano M. The economics of health and health care. New York: Prentice Hall; 2001.

17. Nussbaum M, Sen A. Quality of life. Oxford: Oxford University Press; 1993.

18. Richardson J, McKie J. Empiricism, ethics and orthodox economic theory: what is the appropriet basis for decision-making in the health sector? Soc Sci Med 2005; 60:265-75.

19. Birch S, Gafni A. Information Creat to Evade Reality (ICER): things we should not look to for answers. Pharmaeconomics 2006; 24:1121-31.
20. Nord E, Richardson J, Macarounas-Kirchmann K. Social evaluation of health care versus personal evaluation of health states: evidences on the validity of four health state scaling instruments using Norwegian and Australian survey data. West Heidelberg: Centre for Health Program Evaluation; 1992. (Working Paper, 23).

21. Olsen JA. A note on eliciting distributive preferences for health. J Health Econ 2000; 19:541-50.

22. Dolan P, Tsuchiya A, Armitage C, Brazier J, Bryan $\mathrm{S}$, Eiser D, et al. What is the value to society of a QALY? London: National Institute for Clinical Excellence/National Co-ordinating Centre for Research Metholdology; 2003.

23. Krauss-Silva L. Avaliação tecnológica em saúde: questões metodológicas e operacionais. Cad Saúde Pública 2004; 20 Suppl 2:S199-207.

24. Luce BR, Manning WG, Siegel JE, Lipscomp J. Estimating cost in cost-effectiveness analysis. In: Gold MR, Siegel JE, Russel LB, Weinstein MC, editors. Cost-effectiveness in health and medicine. New York: Oxford University Press; 1996. p. 176-213.

25. Koopmanschap MA, Rutten FF, van Ineveld BM, van Roijen $L$. The friction cost method for measuring indirect costs of disease. J Health Econ 1995; 14:171-89.

26. Meltzer D. Accounting for future costs in medical cost-effectiveness analysis. J Health Econ 1997; 16:33-64.

27. Weinstein MC, Fineberg HV. Clinical decision analysis. In: Weinstein MC, Fineberg HV, Elstein AS, Frazier HS, Neuhauser D, Neutra RR, et al., editors. Clinical decision analysis. Philadelphia: WB Saunders Company; 1980. p. 84-94.

28. Weinstein MC. Economic assessment of medical practices and technologies. Med Decis Making 1981; 1:309-30.

29. Drummond MF, O'Brien BJ, Stoddart G. Methods for economic evaluation of health care programmes. Oxford: Oxford University Press; 1997.

30. Mushlin AI, Fintor L. Is screening for breast cancer cost-effective? Cancer 1992; 69(7 Suppl):1957-62.

31. Lipscomb J. Time preference for health in cost-effectiveness analysis. Med Care 1989; 27(3 Suppl): S233-53.

32. Katz DA, Welch HG. Discouting in cost-effectiveness analysis of healthcare programmes. Pharmacoeconomics 1993; 3:276-85.

33. Lipscomb J, Weinstein MC, Torrance GW. Time preference. In: Gold MR, Siegel JE, Russel LB, Weinstein MC, editors. Cost-effectiveness in health and medicine. New York: Oxford University Press; 1996. p. 214-46.

34. Gravelle H, Smith D. Discouting for health effects in cost benefit and cost effectiveness analysis. Health Econ 2001; 10:587-99.

35. Bos JM, Beutels P, Annemans L, Postma MJ. Valuing prevention economic evaluation: some considerations regardind the choice of discount model for health effects with focus on infectious diseases. Pharmacoeconomics 2004; 22:1171-9.

36. Luce BR. What will it take to make cost-effectiveness analysis acceptable in the United States? Med Care 2005; 43(7 Suppl): 44-8. 
37. Drummond M, Schulper BA. Common methodological flaws in economic evaluation. Med Care 2005; 43(7 Suppl):5-14.

38. Briggs AH, Sculpher MJ, Buxton M. Uncertainty in the economic evaluation of health care technologies: the role of sensitivity analysis. Health Econ 1994; 3:95-104.

39. Rovira J. Evaluación económica en salud: de la investigación a la toma de decisiones. Rev Esp Salud Pública 2004; 78:293-5.

40. Adam T, Evans D, Koopmanschap M. Cost-effectiveness analysis: can we reduce variability in coting methods? Int J Technol Assess Health Care 2003; 19:407-20.

41. Sculpher M, Ades AE, Claxton K. Evidence synthesis, parameter correlation, and probabilistic sensitivity analysis. http://www.hsrc.ac.uk/current_ research/research_programms/mpes.htm (acessado em Abr/2005).

42. Wenseberger J. Dealing with medical practice variation: a proposal for action. Health Aff 1984; 3:6-32.
43. Sancho LG. Como pensar "custo" de forma mais abrangente. Saúde Debate 1999; 23:93.

44. Weinstein M. Recent developments in decisionanalytic modelling for economic evaluation. Pharmacoeconomics 2006; 24:1043-53.

45. Sculpher MJ, Pang FS, Manca A, Drummond MF, Golder S, Urdahl H, et al. Generalisability in economic evaluation studies in healthcare: a review and case studies. Health Technol Assess 2004; 8: iii-iv, 1-192.

46. Vianna CMM, Caetano R. Diretrizes metodológicas para estudos de avaliação econômica de tecnologias para o Ministério da Saúde. http://portal. saude.gov.br/portal/arquivos/pdf/diretrizes_ metodologicas_ave.pdf (acessado em Out/2007).

47. Brouwer WBF, Koopmanschap MA. On the economic foundantion of CEA. Ladies and gentlemen, take your positions! J Health Econ 2000; 19:439-59.

Recebido em 09/Nov/2007

Versão final reapresentada em 06/Ago/2008 Aprovado em 25/Ago/2008 